\title{
EVALUATION OF DEMOGRAPHIC, CLINIC AND GENETIC CHARACTERISTICS OF PATIENTS ADMITTED TO TRAKYA UNIVERSITY HOSPITAL WITH HYPERTROPHIC CARDIOMYOPATHY
}

\author{
Burak Bardakçı $^{1}$ (D), Berfin Tan ${ }^{1}$ (D), Sarper Kızılkaya ${ }^{1}$ (D), ${\text { Ceren } \text { Yüksel }^{1} \text { (D), Servet Altay }}^{2}$ (i)
}

${ }^{1}$ Trakya University School of Medicine, Edirne, TURKEY

${ }^{2}$ Department of Cardiology, Trakya University School of Medicine, Edirne, TURKEY

\begin{abstract}
Aims: This study aims to evaluate the genetics, clinical characteristics, and functional abnormalities of patients diagnosed with hypertrophic cardiomyopathy in Trakya University Hospital. Methods: This retrospective study was conducted with patients who were diagnosed with hypertrophic cardiomyopathy between November 2009 - November 2019 in Trakya University Hospital. The data were obtained from the hospital's database. Patients' data (regarding age, gender, genetics, transthoracic echocardiogram findings, medications, types of hypertrophic cardiomyopathy, and first diagnoses) were examined. Numbers, percentages, means, and standard deviations were used as descriptive statistics. Results: Eleven patients with hypertrophic cardiomyopathy were evaluated. Five (45.45\%) were female and $6(54.54 \%)$ were male. The mean age of the female patients was $58.20 \pm 8.57$ years. The most common type of hypertrophic cardiomyopathy was found to be asymmetrical septal cardiomyopathy [7 (63.63\%)]. Three (27.27\%) patients presented with hypertension. There were gene mutations in three patients. Among these three patients, two (18.18\%) patients have MYBPC3, and one (9.09\%) patient has TTN gene mutations. Conclusion: Hypertrophic cardiomyopathy is usually accompanied by comorbidities such as arrhythmias, myocardial infarction, coronary artery disease. Therefore, these patients must be paid attention to in these matters. Keywords: Hypertrophic cardiomyopathy, hypertension, sudden cardiac death
\end{abstract}

\section{INTRODUCTION}

Defined as the most inheritable group of cardiovascular diseases, cardiomyopathies differ in three major types based on the world's overall population prevalence: hypertrophic (1:500), dilated (1:2500), arrhythmogenic (1:5000) (1). With cases reported from more than 50 countries and having a ratio of 1:500 in the population, hypertrophic cardiomyopathy (HCM) is the most frequent of these three major types (2). Although many patients show either no or minor symptoms and are diagnosed incidentally, they may also display diverse symptoms such as exertional dyspnea, fatigue, unexplained syncope, atypical chest pain, arrhythmia, and sudden cardiac death (SCD) $(1,3,4)$.

The diagnosis is primarily based on echocardiographic imaging of asymmetrical left ventricular hypertrophy without any symptoms of other cardiac or systemic diseases, but the assessments of left ventricular outflow tract gradients, systolic and diastolic function, and myocardial anatomy and function are also used for the diagnosis of HCM (5). Even though any segment of the myocardium can be affected due to HCM, almost $90 \%$ of the cases manifest that the septum is involved (6). According to the European Society of Cardiology guidelines, the diagnostic criteria for HCM is a left ventricular wall thickness of $\geq 15 \mathrm{~mm}$ measured with an echocardiogram or other imaging techniques (7).

Pivoting on different variables such as the reporting era or the source, the annual mortality risk of HCM spans from $<1 \%$ in the general population to $3-6 \%$ in tertiary referral centers $(8,9)$. The reasons for HCM related deaths are varying, but according to the reports, heart failure, stroke, and SCD are the most occurring modes of death (8).

This study aims to examine clinical and genetic characteristics, cardiac structure and functional abnormalities of patients diagnosed with HCM in Trakya University School of Medicine, to assess HCM Risk-SCD score, and to establish if these factors affect the prognosis in the light of literature.

\section{MATERIAL AND METHODS}

This study was approved by the Scientific Research Ethics Committee of Trakya University School of Medicine (Protocol Code: TÜTF-BAEK 2020/440). In this retrospective study, patients who applied to Trakya University School of Medicine, Department of Cardiology and were diagnosed with HCM between November 2009 - November 2019 in Trakya University Hospital were evaluated respectively by their data. The data were obtained from the hospital database. Patients' data were examined regarding age, gender, family history, syncope history, arrhythmia history, septum thickness, apical thickness, ejection fraction, presence of pulmonary hypertension, presence of systolic anterior motion, history of mitral valve disease, presence of left atrial enlargement, genetic results, presence of a pacemaker, and 10-year mortality. Patients older than 18 years were included in the study. 
The data were analyzed using IBM SPSS version 22 (IBM Corp. Released 2013. IBM SPSS Statistics for Windows, Version 22.0. Armonk, NY: IBM Corp.). Numbers, percentages, mean, and standard deviation (SD) were used as descriptive statistics.

\section{RESULTS}

A total of 11 patients with various types of HCM were analyzed. Five $(45.45 \%)$ of them were female and $6(54.54 \%)$ of them were male. The mean age of the female patients was $58.20 \pm 8.57$ years and the mean age of the male patients was $46.67 \pm 6.54$ years. Four (36.36\%) patients presented with chest pain, 3 (27.27\%) patients presented with dyspnea, 3 (27.27\%) patients presented with palpitation, and $1(9.09 \%)$ patient was asymptomatic. Five of the patients were diagnosed with positive troponin and false axis at first. All the first diagnoses are shown in Table 1.

In our study group, the most common type of HCM was found to be asymmetrical septal cardiomyopathy [7 (63.63\%)], followed by apical and midventricular types, respectively [3 (27.27\%), $1(9.09 \%)]$. HCM types of patients and the distribution regarding gender are shown in Table 2.

Table 1: First diagnoses of the patients.

\begin{tabular}{lc} 
First Diagnosis & Number (\%) \\
\hline Screening due to family history & $1(9.09)$ \\
Elevated troponin level and false axis & $5(45.45)$ \\
Clinical consult due to symptoms & $3(27.27)$ \\
ECG/TTE findings and hypertension & $2(18.18)$ \\
\hline
\end{tabular}

ECG/TTE: Electrocardiogram/transthoracic echocardiogram

Table 3: Medications used by the patients.

\begin{tabular}{ll} 
Medication & Number $(\%)$ \\
\hline ACEI/ARBs & $7(63.63)$ \\
Calcium channel blockers & $2(18.18)$ \\
Beta blockers & $11(100)$ \\
Oral anticoagulants & $2(18.18)$ \\
Diuretics & $5(45.45)$ \\
Acetylsalicylic acid & $6(54.54)$ \\
Amiodarone & $2(18.18)$ \\
Statins & $4(36.36)$ \\
Antidiabetics & $1(9.09)$
\end{tabular}

ACEI/ARBs: Angiotensin converting enzyme inhibitor/angiotensin receptor blockers
Beta-blockers were the most frequently used medications, used by all patients in our study group [11 (100\%)], followed by angiotensin-converting enzyme inhibitor/angiotensin receptor blockers (ACEI/ARBs) [7 (63.63\%)]. The distribution of the medications used by patients is shown in Table 3 .

In our study group, arrhythmias [5 (45.45\%)] were found to be the most common comorbidities, followed by coronary artery diseases [4 (36.36\%)]. Atrial fibrillation (AF) and ventricular extrasystole were the most frequent types of arrhythmias with 3 (27.27\%) patients affected. All fifteen comorbidities seen in our patient group are shown in Table 4.

Three (60\%) female and $6(100 \%)$ male patients presented with ischemic findings in the electrocardiogram. Five (45.45\%) of the patients had left ventricle out track obstruction (LVOTO). Transthoracic echocardiogram (TTE) showed systolic anterior movement in three (27.27\%) patients. TTE findings are shown in Table 5.

Data regarding genetic mutations were available only for 5 patients. Among these five patients, two (18.18\%) patients have MYBPC3 and one (9.09\%) patient has TTN gene mutations.

Table 2: Hypertrophic cardiomyopathy types of the patients.

\begin{tabular}{lc} 
HCM Type & Total [n (\%)] \\
\hline Asymmetrical septal & $7(63.64)$ \\
Apical & $3(27.27)$ \\
Midventricular & $1(9.09)$ \\
\hline
\end{tabular}

HCM: Hypertrophic cardiomyopathy

Table 4: Comorbidities of the patients.

\begin{tabular}{ll} 
Comorbidity & Number (\%) \\
\hline Hypertension & $3(27.27)$ \\
Diabetes mellitus & $2(18.18)$ \\
Coronary artery disease & $4(36.36)$ \\
Chronic kidney disease & $3(27.27)$ \\
Myocardial infarction & $2(18.18)$ \\
Cerebrovascular accident & $1(9.09)$ \\
Cardiac abnormality & $1(9.09)$ \\
Atrial fibrillation & $3(27.27)$ \\
Ventricular tachycardia & $1(9.09)$ \\
Anytype of arrhythmia & $5(45.45)$ \\
Chronic liver disease & $1(9.09)$ \\
VEA in Holter & $3(27.27)$ \\
Cardiac arrest & $1(9.09)$ \\
Death & $1(9.09)$ \\
Complications of HCM & $3(27.27)$ \\
\hline VEA Ventricular extasyste
\end{tabular}

VEA: Ventricular extrasystole, HCM: Hypertrophic cardiomyopathy 
Table 5: Transthoracic echocardiogram findings' distribution regarding patients' gender.

\begin{tabular}{|c|c|c|}
\hline TTE Findings & $\begin{array}{c}\text { Female } \\
(\text { mean } \pm S D)\end{array}$ & $\begin{array}{c}\text { Male } \\
(\text { mean } \pm S D)\end{array}$ \\
\hline LV diastolic diameter $(\mathrm{mm})$ & $44 \pm 1.67$ & $45.67 \pm 0.84$ \\
\hline Diastolic IVS thickness ( $\mathrm{mm}$ ) & $16 \pm 2.34$ & $17 \pm 2.66$ \\
\hline$E F(\%)$ & $59 \pm 1.87$ & $60.17 \pm 1.86$ \\
\hline LA diameter $(\mathrm{mm})$ & $36.80 \pm 3.45$ & $42.5 \pm 1.17$ \\
\hline LV systolic diameter $(\mathrm{mm})$ & $26.20 \pm 1.06$ & $24.67 \pm 1.49$ \\
\hline Diastolic posterior wall thickness ( $\mathrm{mm}$ ) & $11.60 \pm 1.02$ & $13.33 \pm 1.83$ \\
\hline Ascending aorta diameter ( $\mathrm{mm})$ & $34.40 \pm 3.32$ & $31 \pm 0.73$ \\
\hline Aortic root $(\mathrm{mm})$ & $21.5 \pm 1.50$ & $20.33 \pm 1.45$ \\
\hline
\end{tabular}

TTE: Transthoracic echocardiogram, SD: Standard deviation, LV: Left ventricle,

IVS: Interventricular septum, EF: Ejection fraction, LA: Left atrium

\section{DISCUSSION}

The mean age of our study was $58.20 \pm 8.57$ years for female and $46.67 \pm 6.54$ years for male patients, which was in line with literature $(10,11)$.

While the studies conducted by van Velzen et al. (10) and Olivotto et al. (11) showed comparable results on the frequencies of symptoms between these studies, [these results being $18 \%$ of patients presenting with chest pain, $14 \%$ presenting with dyspnea, and $8 \%$ presenting with palpitations on the study of van Velzen et al. (10) and $12 \%, 14 \%$, and $8 \%$, respectively in the study of Olivotto et al. (11)], our study showed patients more frequently presenting with symptoms, $36.36 \%$ with chest pain, $27.27 \%$ with dyspnea, and $27.27 \%$ with palpation. It should be noted that both studies included a higher number of patients than our study.

In general, the most common type of hypertrophy is asymmetrical hypertrophy, and it is followed by left ventricular hypertrophy (2). Also, in our study, we found the most common type of hypertrophy as asymmetrical hypertrophy, but in contrast, there were no patients with left ventricular hypertrophy. This may be due to the small number of patients.

Beta-blockers and ACEI/ARBs are the most commonly used medications among our patients. Beta-blockers are the first-line medications of treatment of the HCM (4). ACEI/ARBs are used in many conditions which may accompany such as hypertension, coronary artery disease, chronic kidney disease, or diabetes mellitus $(12,13)$. All these accompanying conditions are common among our patients.

Although it remains ambiguous whether there is a positive association between HTN and HCM, literature shows statistically significant differences between HCM with or without accompanying $\operatorname{HTN}(14,15)$.

Another evaluation was done based on gene mutations. Although gene mutations are thought to be the founding cause of HCM, in our study, only three patients out of 11 were observed to have causative mutations (16). Two (18.18\%) had MYBPC3 gene mutations and one $(9.09 \%)$ had TTN gene mutations. Contrary, in a study by Richard et al. (17), 43\% of the patients showed MYBPC3 gene mutations. Another study also showed MYBPC3 mutations in $39 \%$ of patients (10). Our limited number of patients may cause different results with the literature.
One of HCM's main defining features and one of the biggest factors on its prognosis is LVOTO (18). In our study, LVOTO was observed in $45.45 \%$ of the patients. Maron et al. (19) saw the same factor in $25 \%$ of the patients in their study.

Two (18.18\%) of our patients had a history of myocardial infarction (MI). MI may occur due to myocardial bridging, coronary circulatory insufficiency due to hypertrophy, or high diastolic pressure $(20,21)$. Since serum levels of troponin are elevated in both MI and HCM, these two patients may be misdiagnosed with MI. Also, it may cause the diagnosis of MI to be overlooked.

Atrial fibrillation represents the most common sustained arrhythmia in both general and HCM populations (22). With the reported prevalence of $18-28 \%$, AF appears to be 4 to 6 times more common in patients with HCM (22). In our study, AF was the most common type of arrhythmia with a $27.27 \%$ ratio, in line with the literature.

Sudden cardiac death is the most frequent cause of sudden death among athletes and the most common cause of SCD is HCM among this population (20). There were one ventricular tachycardia and one SCD case in our study. Therefore, HCM patients should be followed up for SCD. Implantable cardioverter-defibrillators should be placed in patients with high HCM risk-SCD score.

The small number of subjects is the main limitation of our study. Moreover, it is a retrospective, single-center study. A multi-center study or a more extensive period of time could have wielded results closer to those of current literature and could have been more illuminating on various connections of different factors of HCM. Further research could include more detailed data on family histories and genetic markers of HCM.

In conclusion, this study provided general information on the clinical and genetic characteristics of HCM. Further studies are needed to overcome the limitations and enlighten more of the nature of HCM, its accompanying factors, and their effects on the diagnosis.

Ethics Committee Approval: This study was approved by the Scientific Research Ethics Committee of Trakya University School of Medicine (Protocol Code: TÜTF-BAEK 2020/440).

Informed Consent: N/A

Conflict of Interest: The authors declared no conflict of interest. 
Author Contributions: Concept: BB, BT, SK, CY, SA. Design: BB, BT, SK, CY, SA. Supervision: BB, BT, SK, CY, SA. Resources: BB, BT, SK, CY, SA. Materials: BB, BT, SK, CY, SA. Data collection and/or processing: BB, BT, SK, CY, SA. Analysis and/or Interpretation: BB, BT, SK, CY, SA. Literature Search: BB, BT, SK, CY, SA. Writing Manuscript: BB, BT, SK, CY, SA. Critical Review: BB, BT, SK, CY, SA.

Financial Disclosure: The authors declared that this study received no financial support.

Editor-in-Chief's Note: Four of the authors of this article, Burak Bardakçı, Berfin Tan, Sarper Kızılkaya, Ceren Yüksel are members of the editorial board of Turkish Medical Student Journal. However, they did not take place at any stage of the editorial decision of the manuscript. The editors who evaluated this manuscript are from other institutions.

\section{REFERENCES}

1. Sabater-Molina M, Pérez-Sánchez I, Hernández Del Rincón JP et al. Genetics of hypertrophic cardiomyopathy: a review of current state. Clin Genet 2018;93(1):314.

2. Maron BJ, Maron MS. Hypertrophic cardiomyopathy. Lancet 2013;381(9862):24255.

3. Cruickshank S. Cardiomyopathy. Nurs Stand 2004;18(23):46-56.

4. Teekakirikul P, Zhu W, Huang HC et al. Hypertrophic cardiomyopathy: an overview of genetics and management. Biomolecules 2019;9(12):878.

5. Wigle ED, The diagnosis of hypertrohic cardiomyopatyh. Heart 2001;86(6):70914.

6. Hensley N, Dietrich J, Nyhan D et al. M. Hypertrophic cardiomyopathy: a review. Anesth Analg 2015;120(3):554-69.

7. Marian AJ, Braunwald E. Hypertrophic cardiomyopathy: genetics, pathogenesis, clinical manifestations, diagnosis, and therapy. Circ Res 2017;121(7):749-70.

8. Shah M. Hypertrophic cardiomyopathy. Cardiol Young 2017;27(S1):25-30.

9. Noureldin RA, Liu S, Nacif MS et al. The diagnosis of hypertrophic cardiomyopathy by cardiovascular magnetic resonance. J Cardiovasc Magn Reson 2012;14(1):17.
10. van Velzen HG, Schinkel AFL, Baart SJ et al. Effect of gender and genetic mutations on outcomes in patients with hypertrophic cardiomyopathy. Am J Cardiol 2018;122(11):1947-54

11. Olivotto I, Maron MS, Adabag AS et al. Gender-related differences in the clinical presentation and outcome of hypertrophic cardiomyopathy. J Am Coll Cardiol 2005;46(3):480-7.

12. Brown NJ, Vaughan DE. Angiotensin converting enzyme inhibitors. Circulation 1990;97(14):1411-20.

13. Cordonnier DJ, Zaoui P, Halimi S. Role of ACE inhibitors in patients with diabetes mellitus. Drugs 2001;61(13):1883-92.

14. Deng T, Ou B, Zhu T et al. The effect of hypertension on cardiac structure and function in different types of hypertrophic cardiomyopathy: a single-center retrospective study. Clin Exp Hypertens 2019;41(4):359-65.

15. Papadopoulos DP, Papademetriou V. Hypertrophic and hypertensive hypertrophic cardiomyopathy--a true association? Angiology 2010;61(1):92-9.

16. Teenkakirikul P, Zhu W, Huang HC et al. Hypertrophic cardiomyopathy; an overview of genetics and management. Biomolecules 2019;19(9):878

17. Richard P, Charron P, Carrier L et al. Hypertrophic cardiomyopathy: distribution of disease genes, spectrum of mutations, and implications for a molecular diagnosis strategy. Circulation 2003;107(17):2227-32.

18. Yacoub MH, El-Hamamsy I, Said $\mathrm{K}$ et al. The left ventricular outflow in hypertrophic cardiomyopathy: from structure to function. J Cardiovasc Transl Res 2009;2(4):510-7.

19. Maron MS, Olivotto I, Betocchi S et al. Effect of left ventricular outflow tract obstruction on clinical outcome in hypertrophic cardiomyopathy. N Engl J Med 2003;348(4):295-303.

20. Lee MS, Chen $\mathrm{CH}$. Myocardial bridging: an up-to-date review. J Invasive Cardiol 2015;27(11):521-8.

21. Raphael CE, Cooper R, Parker $\mathrm{KH}$ et al. Mechanism of myocardial ischemia in hypertrophic cardiomyopathy: insights from wave intensity analysis and magnetic resonance. J Am Coll Cardiol 2016;68(15):1651-60.

22. Maclntyre C, Lakdawala NK. Management of atrial fibrillation in hypertrophic cardiomyopathy. Circulation 2016;133(19):1901-5. 